Supporting Information

\title{
Evidence of Variable H-Bond Network Embedding Nitroxide Free Radicals in Protic Solvents
}

\author{
Michele Pavone, Atte Sillanpää, Paola Cimino, Orlando Crescenzi, Vincenzo Barone*
}

\section{Computational details}

The Car-Parrinello (CP) ${ }^{1}$ approach was used to generate the MD trajectories. The gradient-corrected density functional of Perdew, Burke and Ernzerhof $(\mathrm{PBE})^{2}$ was adopted, the electronic variables being expanded in a plane-wave basis-set up to a 25 Ry cut-off energy. Core states were projected out by pseudo-potentials built according to Vanderbilt's "ultra-soft" scheme. ${ }^{3}$ The time step for integrating the equations of motion was $0.15 \mathrm{fs}$, the fictitious electronic mass was set to 700 a.u., and hydrogens were given the mass of deuterium. These choices have been proven to be effective for structural investigations within the CP scheme. ${ }^{4}$ Periodic boundary conditions were employed, using a rhombic dodecahedral cell that contained the proxyl molecule together with 31 waters, or 23 methanol molecules. Trajectories were collected in the NVT ensemble $(330 \mathrm{~K})$ for a total time of 11.0 and 17.5 ps for methanolic and aqueous solutions, respectively. Starting configurations were extracted from equilibrated classical MD trajectories. For statistical analysis, snapshots were extracted at regular time intervals from the last 5 ps (for methanolic solution) or $7.5 \mathrm{ps}$ (for aqueous solution) of the trajectories.

Quantum-chemical calculations of the interaction energies within nitroxide-solvent clusters were performed by the Gaussian03 suite of programs ${ }^{5}$ employing the reliable hybrid density functional known as PBE0, ${ }^{6}$ and the $6-311++\mathrm{G}(3 \mathrm{df}, 2 \mathrm{pd})$ basis set, ${ }^{7}$ which provides converged nitroxide geometrical parameters. ${ }^{8}$

\section{References}

(1) R. Car, M. Parrinello, Phys. Rev. Lett. 1985, 55, 2471.

(2) J.P. Perdew, K. Burke, M. Ernzerhof, Phys. Rev. Lett. 1996, 77, 3865.

(3) D. Vanderbilt, Phys. Rev. B 1990, 41, 7892.

(4) J.C. Grossman, E. Schwegler, E. W. Draeger, F. Gygi, and G. Galli, J. Chem. Phys. 2004, 120, 300.

(5) Gaussian 03, Revision C.02, Frisch, M. J.; Trucks, G. W.; Schlegel, H. B.; Scuseria, G. E.; Robb, M. A.; Cheeseman, J. R.; Montgomery, Jr., J. A.; Vreven, T.; Kudin, K. N.; Burant, J. C.; Millam, J. M.; Iyengar, S. S.; Tomasi, J.; Barone, V.; Mennucci, B.; Cossi, M.; Scalmani, G.; Rega, N.; Petersson, G. A.; Nakatsuji, H.; Hada, M.; Ehara, M.; Toyota, K.; Fukuda, R.; Hasegawa, J.; Ishida, M.; Nakajima, T.; Honda, Y.; Kitao, O.; Nakai, H.; Klene, M.; Li, X.; Knox, J. E.; Hratchian, H. P.; Cross, J. B.; Bakken, V.; Adamo, C.; Jaramillo, J.; Gomperts, R.; Stratmann, R. E.; Yazyev, O.; Austin, A. J.; Cammi, R.; Pomelli, C.; Ochterski, J. W.; Ayala, P. Y.; Morokuma, K.; Voth, G. A.; Salvador, P.; Dannenberg, J. J.; Zakrzewski, V. G.; Dapprich, S.; Daniels, A. D.; Strain, M. C.; Farkas, O.; Malick, D. K.; Rabuck, A. D.; Raghavachari, K.; Foresman, J. B.; Ortiz, J. V.; Cui, Q.; Baboul, A. G.; Clifford, S.; Cioslowski, J.; Stefanov, B. B.; Liu, G.; Liashenko, A.; Piskorz, P.; Komaromi, I.; Martin, R. L.; Fox, D. J.; Keith, T.; Al-Laham, M. A.; Peng, C. Y.; Nanayakkara, A.; Challacombe, M.; Gill, P. M. W.; Johnson, B.; Chen, W.; Wong, M. W.; Gonzalez, C.; and Pople, J. A.; Gaussian, Inc., Wallingford CT, 2004.

(6) C. Adamo, V. Barone, J. Chem. Phys. 1999, 110, 6158.

(7) M. J. Frisch, J. A. Pople, J. S. Binkley, J. Chem. Phys. 1984, 80, 3265.

(8) Pavone, M.; Cimino, P.; De Angelis, F.; Barone, V.; J. Am. Chem. Soc. 2006, 128, 4338. 


\section{Cartesian coordinates (Angstroms)}

\section{PROXYL- $\left(\mathrm{H}_{2} \mathrm{O}\right)_{2}$}

\begin{tabular}{lrrr}
$\mathrm{N}$ & -0.000057 & -0.034741 & -0.002470 \\
$\mathrm{O}$ & 0.007370 & 1.228461 & -0.004454 \\
$\mathrm{C}$ & -1.239566 & -0.817751 & -0.195343 \\
$\mathrm{C}$ & 1.229969 & -0.831521 & 0.195325 \\
$\mathrm{C}$ & -2.002752 & -0.304028 & -1.405722 \\
$\mathrm{H}$ & -2.363520 & 0.711511 & -1.244360 \\
$\mathrm{H}$ & -2.866346 & -0.946523 & -1.586248 \\
$\mathrm{H}$ & -1.373312 & -0.315617 & -2.297100 \\
$\mathrm{C}$ & -2.097761 & -0.715802 & 1.064983 \\
$\mathrm{H}$ & -3.021709 & -1.279460 & 0.923774 \\
$\mathrm{H}$ & -2.359433 & 0.323619 & 1.265362 \\
$\mathrm{H}$ & -1.577990 & -1.121713 & 1.934683 \\
$\mathrm{C}$ & 2.092612 & -0.741389 & -1.062816 \\
$\mathrm{H}$ & 1.570094 & -1.142077 & -1.933275 \\
$\mathrm{H}$ & 3.009309 & -1.315960 & -0.918469 \\
$\mathrm{H}$ & 2.367345 & 0.294535 & -1.263839 \\
$\mathrm{C}$ & 1.995640 & -0.324122 & 1.406876 \\
$\mathrm{H}$ & 2.368038 & 0.687077 & 1.244657 \\
$\mathrm{H}$ & 1.363742 & -0.327109 & 2.296585 \\
$\mathrm{H}$ & 2.851572 & -0.975830 & 1.590844 \\
$\mathrm{C}$ & 0.640453 & -2.229246 & 0.395266 \\
$\mathrm{H}$ & 1.329661 & -3.005400 & 0.062626 \\
$\mathrm{H}$ & 0.444289 & -2.396253 & 1.456995 \\
$\mathrm{C}$ & -0.666150 & -2.222245 & -0.394298 \\
$\mathrm{H}$ & -0.471919 & -2.392304 & -1.455943 \\
$\mathrm{H}$ & -1.364012 & -2.990311 & -0.060922 \\
$\mathrm{H}$ & 1.535352 & 2.287242 & -0.248516 \\
$\mathrm{O}$ & 2.385941 & 2.738414 & -0.362418 \\
$\mathrm{H}$ & 2.184033 & 3.549518 & -0.828048 \\
$\mathrm{H}$ & -1.508377 & 2.304930 & 0.243792 \\
$\mathrm{O}$ & -2.354499 & 2.762490 & 0.365093 \\
$\mathrm{H}$ & -2.141583 & 3.575013 & 0.823291 \\
& & & \\
\hline & &
\end{tabular}




\section{PROXYL- $\left(\mathrm{CH}_{3} \mathrm{OH}\right)$}

$\begin{array}{lrrr}\mathrm{N} & 0.317785 & -0.244374 & 0.107881 \\ \mathrm{O} & -0.687013 & -0.970537 & 0.334175 \\ \mathrm{C} & 1.666744 & -0.824438 & -0.044070 \\ \mathrm{C} & 0.248845 & 1.229671 & 0.013951 \\ \mathrm{C} & 1.623214 & -1.971540 & -1.039438 \\ \mathrm{H} & 0.963113 & -2.760530 & -0.678925 \\ \mathrm{H} & 2.623705 & -2.386671 & -1.172841 \\ \mathrm{H} & 1.255849 & -1.633158 & -2.009544 \\ \mathrm{C} & 2.147302 & -1.319592 & 1.319039 \\ \mathrm{H} & 3.119917 & -1.805057 & 1.220809 \\ \mathrm{H} & 1.436659 & -2.042303 & 1.721704 \\ \mathrm{H} & 2.242332 & -0.498582 & 2.031757 \\ \mathrm{C} & -0.404433 & 1.618455 & -1.311861 \\ \mathrm{H} & 0.176661 & 1.258688 & -2.163095 \\ \mathrm{H} & -0.477437 & 2.705472 & -1.381558 \\ \mathrm{H} & -1.411251 & 1.204053 & -1.376277 \\ \mathrm{C} & -0.541169 & 1.795251 & 1.183070 \\ \mathrm{H} & -1.584216 & 1.481657 & 1.140425 \\ \mathrm{H} & -0.115925 & 1.469878 & 2.134014 \\ \mathrm{H} & -0.511105 & 2.885850 & 1.147316 \\ \mathrm{C} & 1.735110 & 1.593629 & 0.058220 \\ \mathrm{H} & 1.939505 & 2.516824 & -0.484266 \\ \mathrm{H} & 2.043486 & 1.746614 & 1.095271 \\ \mathrm{C} & 2.457288 & 0.387609 & -0.538800 \\ \mathrm{H} & 2.425392 & 0.425522 & -1.630139 \\ \mathrm{H} & 3.505235 & 0.335434 & -0.242548 \\ \mathrm{H} & -2.418549 & -0.397189 & 0.090642 \\ \mathrm{O} & -3.291563 & -0.011286 & -0.083478 \\ \mathrm{C} & -4.261908 & -1.021569 & -0.010284 \\ \mathrm{H} & -5.232676 & -0.568172 & -0.215508 \\ \mathrm{H} & -4.095034 & -1.813918 & -0.750216 \\ \mathrm{H} & -4.307511 & -1.484063 & 0.983265\end{array}$


DTBN- $\left(\mathrm{H}_{2} \mathrm{O}\right)$

$\begin{array}{lrrr}\mathrm{N} & 0.045175 & -0.164444 & -0.150590 \\ \mathrm{O} & -0.837577 & -1.067718 & -0.032034 \\ \mathrm{C} & 1.441513 & -0.694113 & -0.012865 \\ \mathrm{C} & -0.407394 & 1.259376 & -0.010170 \\ \mathrm{C} & -1.927740 & 1.273489 & -0.121477 \\ \mathrm{H} & -2.266404 & 0.843037 & -1.063272 \\ \mathrm{H} & -2.404352 & 0.726553 & 0.688740 \\ \mathrm{H} & -2.259434 & 2.311844 & -0.077555 \\ \mathrm{C} & -0.018584 & 1.814552 & 1.361628 \\ \mathrm{H} & -0.435235 & 1.194858 & 2.156724 \\ \mathrm{H} & -0.428959 & 2.820636 & 1.468056 \\ \mathrm{H} & -0.023694 & 1.664263 & -2.107190 \\ \mathrm{C} & 0.152497 & 2.130631 & -1.136714 \\ \mathrm{H} & 1.059483 & 1.882961 & 1.501379 \\ \mathrm{H} & 1.213715 & 2.339869 & -1.036145 \\ \mathrm{H} & -0.370526 & 3.088263 & -1.127041 \\ \mathrm{C} & 1.556388 & -1.874081 & -0.977805 \\ \mathrm{H} & 0.820972 & -2.639991 & -0.744276 \\ \mathrm{H} & 1.400706 & -1.547171 & -2.007439 \\ \mathrm{H} & 2.555729 & -2.305334 & -0.901157 \\ \mathrm{H} & 0.821301 & -1.877066 & 1.685087 \\ \mathrm{H} & 2.576762 & -1.712556 & 1.514502 \\ \mathrm{H} & 1.623674 & -0.362650 & 2.136875 \\ \mathrm{H} & 3.493355 & -0.194315 & -0.220520 \\ \mathrm{H} & 2.536096 & 1.187463 & 0.277922 \\ \mathrm{H} & 2.480312 & 0.621972 & -1.403332 \\ \mathrm{C} & 2.534299 & 0.306816 & -0.361888 \\ \mathrm{C} & 1.623884 & -1.187912 & 1.424129 \\ \mathrm{H} & -2.708898 & -1.403834 & -0.045733 \\ \mathrm{O} & -3.661113 & -1.574626 & -0.053852 \\ \mathrm{H} & -3.740488 & -2.521492 & 0.056506\end{array}$

